\title{
OPERATOR OF INVARIANT DIFFERENTIATION AND ITS APPLICATION FOR INTEGRATING SYSTEMS OF ORDINARY DIFFERENTIAL EQUATIONS
}

\author{
R.K. GAZIZOV, A.A. GAINETDINOVA
}

\begin{abstract}
We propose an algorithm for integrating $n$-th order ordinary differential equations (ODE) admitting $n$-dimensional Lie algebras of operators. The algorithm is based on invariant representation of the equations by the invariants of the admitted Lie algebra and application of an operator of invariant differentiation of special type. We show that in the case of scalar equations this method is equivalent to the known order reduction methods. We study an applicability of the suggested algorithm to the systems of $m k$ th order ODEs admitting $k m$-dimensional Lie algebras of operators. For the admitted Lie algebra we obtain a condition ensuring the possibility to construct the operator of invariant differentiation of a special type and to reduce the order of the considered system of ODEs. This condition is the implication of the existence of nontrivial solutions to the systems of linear algebraic equations, where the coefficients are the structural constants of the Lie algebra. We present an algorithm for constructing the $(k m-1)$-dimensional Lie algebra for the reduced system. The suggested approach is applied for integrating the systems of two second order equations.
\end{abstract}

Keywords: ordinary differential equations, Lie algebras of operators, differential invariants, operator of invariant differentiation.

Mathematics Subject Classification: 34A25, 22E05

\section{INTRODUCTION}

Group analysis provides a wide set of tools for studying symmetry properties of differential equations, for reducing the orders and for integrating these equations by quadratures, see [1]-[4] and others.

In integrating ordinary differential equations (ODEs), the most used methods are the method of successive order reduction involving the introduction of so-called canonical variables or the introduction of differential invariants, see, for instance, [4, 5]. In the first method, at the first step the equation is transformed to some canonical form and then it is reduced to an equation of smaller order. The second method is based on applying differential invariants of the admitted group and the invariant differentiation operation, that is, the operation of differentiating one differential invariant w.r.t. another invariant of lower order.

The classical theory of differential invariants was founded by S. Lie [1] and was developed in works by A. Tresse [6] and L.V. Ovsyannikov [2]. An important notion of this theory is operator of invariant differentiation (OID), the linear differential operator, the action of which on an arbitrary differential invariant is again a differential invariant, as a rule, of higher order.

R.K. Gazizov, A.A. Gainetdinova, Operator of invariant differentiation and its application FOR INTEGRATING SYSTEMS OF ORDINARY DIFFERENTIAL EQUATIONS.

(C)Gazizov R.K., Gainetdinova A.A. 2017.

The work was made under the support of the Ministery of Education and Science of Russian Federation in the framework of state task no. 1.3103.2017/4.6.

Submitted October 2, 2017. 
Various approaches for constructing OIDs were considered in works [2], [7], 8]. In a series of works OIDs were employed for constructing a basis of differential invariants of the admitted algebra in the problems on classification of differential equations, see, for instance, [9]-[11].

In work [12] there was proposed a method for integrating a system of two second order ODEs with four symmetries; that method was a version of method of successive order reduction. The method employs the result on the classification of systems of two second order ODEs with four symmetries and it is based on employing OIDs for constructing first integrals for these systems. At that, the result of the classification of systems of second order ODEs was used for proving that the considered systems have OID in a form allowing one to employ it for obtaining the first integral of the system.

In the present work the method proposed in [12] is generalized for differential equations of arbitrary form. In particular, there was shown that for the scalar equations, this method is equivalent to the known methods of order reduction. We study the applicability of this method to the systems of $m$ ODEs of $k$ th order admitting $k m$-dimensional Lie algebra of operators. We obtain a condition for the admitted Lie algebra ensuring the possibility of constructing OIDs in the form allowing to use it for order reducing of the considered system of ODEs. We also show that the constructed in this way reduced system admits $(k m-1)$-dimensional Lie algebra and the proposed method can be again employed for this method.

\section{CONSTRUCTING OF OPERATOR OF INVARIANT DiFFERENTIATION}

We consider the system

$$
u^{(k)}=f\left(t, u, u^{(1)}, \ldots, u^{(k-1)}\right)
$$

of $m$ ordinary differential equations of $k$ th order admitting the $n=k m$-dimensional Lie algebra $L_{n}$ generated by the basis operators

$$
X_{i}=\tau_{i} \frac{\partial}{\partial t}+\sum_{\alpha=1}^{m} \xi_{i}^{\alpha} \frac{\partial}{\partial u_{\alpha}}, i=1, \ldots, n .
$$

Here $t$ is an independent variable, $u=\left(u_{1}, \ldots, u_{m}\right)$ is a vector of dependent variables, $u^{(k)}$ is the vector of the derivatives of $k$ th order, $\tau_{i} \xi_{i}^{\alpha}$ are given functions of $t, u, f=\left(f_{1}, \ldots, f_{m}\right)$ is the vector function of the mentioned variables.

Differential invariants of the algebra $L_{n}$ are sought of the system

$$
X_{i}^{(k)} I=0, \quad i=1, \ldots, n,
$$

where $X_{i}^{(k)}$ is obtained from the operator $X_{i}$ by extending to all derivatives of $k$ th order, and the functions $I=I\left(t, u, u^{(1)}, \ldots, u^{(k)}\right)$ are the unknowns. We introduce the matrix

$$
\Omega^{(k)}=\left\|\begin{array}{ccccccc}
\tau_{1} & \xi_{1}^{1} & \ldots & \xi_{1}^{m} & \zeta_{1}^{1(1)} & \ldots & \zeta_{1}^{m(k)} \\
\vdots & & & & & & \\
\tau_{n} & \xi_{n}^{1} & \ldots & \xi_{n}^{m} & \zeta_{n}^{1(1)} & \ldots & \zeta_{n}^{m(k)}
\end{array}\right\|
$$

formed by the coordinates of the extended operators $X_{i}^{(k)}$ and let

$$
\operatorname{rank} \Omega^{(k)}=n \text {. }
$$

Then system (2) has $m+1$ functional independent solutions. If system (1) defines a regular manifold w.r.t the transformation group generated by the operators of the algebra $L_{n}$, then it can be represented in the invariant form (see, for instance, [2], 3]). In this case solution (2) determines $m$ independent invariants $I_{\alpha}^{(k)}$ of order $k(\alpha=1, \ldots, m)$ and one invariant $I$ of smaller order.

The order of the invariant $I$ is defined as follows: if for a $l$ th extension $(l=0, \ldots, k-1)$ the rank of the matrix $\Omega^{(l)}$ is less than $(l+1) m+1$, then the order of $I$ is equal to $l$. In the general 
case, $\operatorname{rank} \Omega^{(l)}$ is equal to $m(l+1)+1$ and the condition $\operatorname{rank} \Omega^{(k)}=n$ implies that the above inequality can hold for an only $l$.

Assume that equation (1) possesses the following invariant representation:

$$
I_{\alpha}^{(k)}=F_{\alpha}(I), \quad \alpha=1, \ldots, m,
$$

where $F_{\alpha}$ are some functions. Let us construct OID $\lambda D_{t}$, where $\lambda=\lambda\left(t, u_{1}, \ldots, u_{m}, \ldots, u_{1}^{(k)}, \ldots, u_{m}^{(k)}\right)$. According [2], the function $\lambda$ can be found by the system of equations

$$
X_{i}^{(k)}(\lambda)-\lambda D_{t}\left(\tau_{i}\right)=0 .
$$

We apply the constructed OID to an invariant of smaller order:

$$
\lambda D_{t}(I)=\Theta\left(I, I_{1}^{(k)}, \ldots, I_{m}^{(k)}\right),
$$

where $\Theta=\Theta\left(I, I_{1}^{(k)}, \ldots, I_{m}^{(k)}\right)$ is some function. Considering the obtained expression on a solution of system (3), we get

$$
\left.\lambda D_{t}(I)\right|_{[3}=\hat{\Theta}(I),
$$

where $\hat{\Theta}(I)=\Theta\left(I, F_{1}(I), \ldots, F_{m}(I)\right)$. Equation (5) can be rewritten as

$$
\frac{\mathrm{d} I}{\hat{\Theta}(I)}=\frac{\mathrm{d} t}{\lambda}
$$

We observe that the left hand side of equation (6) is integrable by quadratures only if the function $\lambda$ can be represented as

$$
\lambda=\frac{1}{D_{t}(\Phi)}
$$

with some function $\Phi=\Phi\left(t, u_{1}, \ldots, u_{m}, \ldots, u_{1}^{(k-1)}, \ldots, u_{m}^{(k-1)}\right)$.

Let us show that the function $\lambda$ for OID can be constructed in as (7). Substituting expression (7) into (4), we obtain

$$
-\frac{1}{\left(D_{t} \Phi\right)^{2}}\left(X_{i}^{(k)}\left(D_{t} \Phi\right)+D_{t} \Phi D_{t}\left(\xi_{i}\right)\right)=0,
$$

which implies (see, for instance, [2])

$$
D_{t}\left(X_{i}^{(k-1)} \Phi\right)=0 .
$$

Thus, the function $\Phi$ should satisfy the system

$$
X_{i}^{(k-1)} \Phi=C_{i}, \quad i=1, \ldots, n
$$

with some constants $C_{i}$. System $(8)$ is a system of first order linear inhomogeneous partial differential equations. It is solvable if and only if it is compatible and complete.

We study the compatibility of system (8) constructed for the operators admitted by system of ODEs 1 . We consider the matrix $\Omega^{(k-1)}$ formed by the coordinates of the operators $X_{i}^{(k-1)}$. This is a matrix of size $n \times(n+1)$ and its rank is equal $n$. Otherwise, if rank $\Omega^{(k-1)}<n$, system (1) has no invariant representation since the admitted group has more than one invariant of lower order and therefore less than $m$ invariants of $k$ th order. Therefore, system (8) is compatible for all $C_{i}$.

In order to study the completeness of system (8), we introduce the operators

$$
Y_{i}=X_{i}^{(k-1)}+C_{i} \frac{\partial}{\partial \Phi} .
$$


According the general method of studying linear inhomogeneous equations, see, for instance, [13], the completeness of system (8) is equivalent to the closedness of the operators $\left\{Y_{i}\right\}$ w.r.t. the commutator. We have

$$
\begin{aligned}
{\left[Y_{i}, Y_{j}\right] } & =\left[X_{i}^{(k-1)}+C_{i} \frac{\partial}{\partial \Phi}, X_{j}^{(k-1)}+C_{j} \frac{\partial}{\partial \Phi}\right]=\left[X_{i}^{(k-1)}, X_{j}^{(k-1)}\right] \\
& =\sum_{s=1}^{n} c_{i j}^{s} X_{s}^{(k-1)}=\sum_{s=1}^{n} c_{i j}^{s}\left(Y_{s}-C_{s} \frac{\partial}{\partial \Phi}\right)
\end{aligned}
$$

This implies that the system of the operators $\left\{Y_{i}\right\}$ is closed provided the constants $C_{s}$ satisfy the system of algebraic equations

$$
\sum_{s=1}^{n} c_{i j}^{s} C_{s}=0
$$

System (9) has the trivial solution $C_{s} \equiv 0, s=1, \ldots, n$, only if $n \geqslant 3$ and the rank of system (9) is equal $n$. In this case the function $\Phi$ obtained from system (8) is an invariant of the admitted Lie algebra, that is, $\Phi=\Phi(I)$, and this is why the obtained operator is not OID and the action on the invariant $I$ does not lead to new invariants; the order of the differential invariant is not increased.

If $n<3$ or $n \geqslant 3$ and the rank of system $(9)$ is less than $n$, then equation (9) has a non-zero solution $\left(C_{1}^{0}, \ldots, C_{n}^{0}\right)^{T}$, where at least one constant $C_{i}^{0} \neq 0$. As the function $\Phi$ for OID, we can choose each particular solution of system $(9)$ with $C_{i}=C_{i}^{0}$. Such function $\Phi$ is invariant w.r.t. linear combinations of form $C_{j}^{0} X_{i}-C_{i}^{0} X_{j}$ :

$$
\left(C_{j}^{0} X_{i}-C_{i}^{0} X_{j}\right)(\Phi)=C_{j}^{0} X_{i}(\Phi)-C_{i}^{0} X_{j}(\Phi)=C_{j}^{0} C_{i}^{0}-C_{i}^{0} C_{j}^{0}=0 .
$$

We are going to show that among these linear combinations, there exist $n-1$ linear independent ones forming a Lie algebra.

Let $C_{1}^{0} \neq 0$ and consider the operators $\hat{X}_{i}=C_{i}^{0} X_{1}-C_{1}^{0} X_{i}, i=2, \ldots, n$.

1. By construction, these operators are linearly independent, since the operators $X_{1}, \ldots, X_{n}$ form the basis of the Lie algebra $L_{n}$.

2. All other combinations can be expressed in terms of the chosen ones:

$$
\frac{1}{C_{1}^{0}}\left(C_{j}^{0} X_{k}-C_{k}^{0} X_{j}\right)=\frac{C_{j}^{0}}{C_{1}^{0}}\left(C_{k}^{0} X_{1}-\hat{X}_{k}\right)-\frac{C_{k}^{0}}{C_{1}^{0}}\left(C_{j}^{0} X_{1}-\hat{X}_{j}\right)=\frac{C_{k}^{0}}{C_{1}^{0}} \hat{X}_{j}-\frac{C_{j}^{0}}{C_{1}^{0}} \hat{X}_{k} .
$$

3. Let us show that the set of the operators $\left\{\hat{X}_{i}\right\}$ is closed w.r.t. the commutation. We have

$$
\begin{aligned}
{\left[\hat{X}_{i}, \hat{X}_{j}\right] } & =\left[C_{i}^{0} X_{1}-C_{1}^{0} X_{i}, C_{j}^{0} X_{1}-C_{1}^{0} X_{j}\right]=C_{1}^{0}\left(C_{j}^{0}\left[X_{1}, X_{i}\right]-C_{i}^{0}\left[X_{1}, X_{j}\right]+C_{1}^{0}\left[X_{i}, X_{j}\right]\right) \\
& =C_{1}^{0} \sum_{r=1}^{n}\left(C_{j}^{0} c_{1 i}^{r}-C_{i}^{0} c_{1 j}^{r}+C_{1}^{0} c_{i j}^{r}\right) X_{r} \\
& =C_{1}^{0}\left(C_{j}^{0} c_{1 i}^{1}-C_{i}^{0} c_{1 j}^{1}+C_{1}^{0} c_{i j}^{1}\right) X_{1}+\sum_{r=2}^{n}\left(C_{j}^{0} c_{1 i}^{r}-C_{i}^{0} c_{1 j}^{r}+C_{1}^{0} c_{i j}^{r}\right)\left(C_{r}^{0} X_{1}-\hat{X}_{r}\right) \\
& =\sum_{r=1}^{n}\left(C_{j}^{0} c_{1 i}^{r}-C_{i}^{0} c_{1 j}^{r}+C_{1}^{0} c_{i j}^{r}\right) C_{r}^{0} X_{1}-\sum_{r=2}^{n}\left(C_{j}^{0} c_{1 i}^{r}-C_{i}^{0} c_{1 j}^{r}+C_{1}^{0} c_{i j}^{r}\right) \hat{X}_{r} \\
& =-\sum_{r=2}^{n}\left(C_{j}^{0} c_{1 i}^{r}-C_{i}^{0} c_{1 j} r+C_{1}^{0} c_{i j}^{r}\right) \hat{X}_{r}
\end{aligned}
$$


where the first sum in the pre-last line vanishes due to (9). Then

$$
\left[\hat{X}_{i}, \hat{X}_{j}\right]=\sum_{s=1}^{n} \hat{c}_{i j}^{s} \hat{X}_{s}, \quad \text { where } \quad \hat{c}_{i j}^{s}=C_{j}^{0} c_{1 i}^{s}-C_{i}^{0} c_{1 j}^{s}+C_{1}^{0} c_{i j}^{s} .
$$

Thus, the operators $\hat{X}_{i}$ generate $(n-1)$-dimensional Lie algebra $L_{n-1}$. Apart of the invariants of the algebra $L_{n}$, the reduced algebra $L_{n-1}$ has an additional invariant, the function $\Phi$.

We return back to integrating equation (6). It can be rewritten in the integrable form

$$
\frac{D_{t}(I)}{\hat{\Theta}(I)}=D_{t}(\Phi)
$$

and its solution

$$
H(\Phi, I)=0
$$

with some function $H$ is the first integral for system of equations (3). Adding this equation to system (3) and removing the differential implications, we arrive at a system of order $k m-1$ admitting the above constructed Lie algebra $L_{n-1}$.

Thus, we have proved the following theorem.

Theorem 1. Suppose that system of $m$ ODEs of $k$ th order (1) admits the $n$-dimensional $(n=k m)$ Lie algebra $L_{n}$ of the operators $X_{i}, i=1, \ldots, n$, and is represented in terms of the differential invariants $I^{(l)}, I_{1}^{(k)}, \ldots, I_{m}^{(k)}$ of this algebra as $(3) ; l$ and $k$ are the orders of the differential invariants. Assume that system of linear algebraic equations (9)

$$
\sum_{s=1}^{n} c_{i j}^{s} C_{s}=0
$$

where $c_{i j}^{s}$ are the structural constants of Lie algebra $L_{n}$, has a non-trivial solution. Then there exists a OID of form

satisfying the relation

$$
\frac{1}{D_{t}(\Phi)} D_{t}
$$

$$
\left.\frac{1}{D_{t}(\Phi)} D_{t}\left(I^{(l)}\right)\right|_{\sqrt[3]{ })}=\hat{\Theta}\left(I^{(l)}\right)
$$

which is integrable and generates the first integral of system (1). The system of order $n-1$ obtained by system (3) by adding the first integral admits $(n-1)$-dimensional Lie algebra $L_{n-1}$ with basis operators constructed as linear combinations of the operators $X_{i}$ with the coefficients determined by the solution of system (9).

Remark. This theorem can be generalized to an arbitrary system of ODEs of nth order admitting $n$ operators. As a demonstration, see Example 3.

\section{INTEGRATION OF SCALAR ODEs}

3.1. First order equation. We consider the applying OID for integrating the first order equation

$$
\dot{x}=f(t, x)
$$

admitting one operator

$$
X=\tau(t, x) \frac{\partial}{\partial t}+\xi(t, x) \frac{\partial}{\partial x} .
$$

Such operator has one independent invariant $I^{(0)}(t, x)$ of zero order and one invariant $I^{(1)}(t, x, \dot{x})$ of first order, while equation (10) has an invariant representation:

$$
I^{(1)}=F\left(I^{(0)}\right) \text {. }
$$


Let us calculated OID. Let $\lambda=\left(D_{t} \Phi\right)^{-1}$, where $\Phi=\Phi(t, x)$ is determined by the equation

$$
X(\Phi) \equiv \tau \Phi_{t}+\xi \Phi_{x}=1 .
$$

Applying OID to the invariant $I^{(0)}$ and taking into consideration 11 , we arrive at the relation:

$$
\left.\frac{D_{t}\left(I^{(0)}\right)}{D_{t}(\Phi)}\right|_{11}=\alpha\left(I^{(0)}\right)
$$

with some function $\alpha$. Integration this equation leads to the solution of equation (11).

We consider equation (10) in an equivalent form

$$
M(t, x) \mathrm{d} t+N(t, x) \mathrm{d} x=0 .
$$

By system of the characteristic equations

$$
\frac{\mathrm{d} t}{\tau}=\frac{\mathrm{d} x}{\xi}=\frac{\mathrm{d} \Phi}{1}
$$

for $(12)$ and by (14) we have

$$
\frac{M(x, y)}{\tau M+\xi N} \mathrm{~d} t+\frac{N(x, y)}{\tau M+\xi N} \mathrm{~d} x=\mathrm{d} \Phi,
$$

which is equivalent to multiplying equation 14 by the integrating factor $\mu=\frac{1}{\tau M+\xi N}$, see, for instance, [4].

Thus, the function $\Phi$ is the total differential obtained for equation (14).

On the other hand, the constructed $\Phi$ and $I^{(0)}$ can be interpreted as new variables. Then equation (13) admits the translation operator $\frac{\partial}{\partial \Phi}$. Therefore, seeking $\Phi$ is equivalent to constructing a new dependent variable while transformation the admitted operator to the translation operator.

Example 1. We consider the equation

$$
\dot{x}+x^{2}=\frac{2}{t^{2}}
$$

admitting the operator $X=t \frac{\partial}{\partial t}-x \frac{\partial}{\partial x}$. We rewrite equation 15 in the equivalent form

$$
\mathrm{d} x+\left(x^{2}-\frac{2}{t^{2}}\right) \mathrm{d} t=0 .
$$

The invariants of the operator $X$ are of the form $I^{(0)}=t x, I^{(1)}=t^{2} \dot{x}$. The function $\Phi$ of OID OID $\left(D_{t} \Phi\right)^{-1} D_{t}$ is determined by the equation $X \Phi=1$ and hence, $\Phi=\ln t+\phi(t x)$, where $\phi(t x)$ is an arbitrary function of the invariant of the operator $X$. We choose $\Phi=\ln t$. Then OID is of the form $t D_{t}$.

We apply the obtained OID to the invariant $I^{(0)}$ :

$$
\left.t D_{t}\left(I^{(0)}\right)\right|_{[15]}=I^{(0)}+2-\left(I^{(0)}\right)^{2}
$$

to obtain

$$
\frac{\mathrm{d} I^{(0)}}{2+I^{(0)}-\left(I^{(0)}\right)^{2}}=\frac{\mathrm{d} t}{t} .
$$

In the initial variables this reads as

$$
\frac{\mathrm{d}(t x)}{2+t x-(t x)^{2}}=\mathrm{d}(\ln t) .
$$

It is easy to see that constructing of this equation is equivalent to multiplying equation (16) by the integrating factor $\mu=\frac{t}{t^{2} x^{2}-t x-2}$. 
3.2. Second order equation. We apply the considered algorithm for integrating the second order differential equation

$$
\ddot{x}=f(t, x, \dot{x})
$$

admitting the Lie algebra $L_{2}$ with the basis operators

$$
X_{i}=\tau_{i}(t, x) \frac{\partial}{\partial t}+\xi_{i}(t, x) \frac{\partial}{\partial x}, \quad i=1,2 .
$$

For example, we consider the case, when the commutator of the admitted operators $\left[X_{1}, X_{2}\right]=$ $X_{1}$ and the operators $X_{1}$ and $X_{2}$ are not linearly connected, that is,

$$
X_{1} \vee X_{2} \equiv \tau_{1} \xi_{2}-\tau_{2} \xi_{1} \neq 0,
$$

see, for instance, [4]. Then the algebra $L_{2}$ has two differential invariants $I^{(1)}(t, x, \dot{x})$ and $I^{(2)}(t, x, \dot{x}, \ddot{x})$ and this allows us to rewrite equation $(19)$ as

$$
I^{(2)}=F\left(I^{(1)}\right)
$$

with some function $F$.

We construct OID as $\left(D_{t} \Phi\right)^{-1} D_{t}$. The completeness of corresponding system (8) implies that the function $\Phi(t, x)$ can be found by the system of equations

$$
\begin{aligned}
& X_{1}(\Phi) \equiv \tau_{1} \Phi_{t}+\xi_{1} \Phi_{x}=0, \\
& X_{2}(\Phi) \equiv \tau_{2} \Phi_{t}+\xi_{2} \Phi_{x}=1 .
\end{aligned}
$$

If $I_{0}$ is an algebraic invariant of the operator $X_{1}$, the general solution to first equation in the above system is written as $\Phi=\phi\left(I_{0}\right)$, while by the condition $\left[X_{1}, X_{2}\right]=X_{1}$ the second equation determines the function $\phi$. Thus, the function $\Phi$ is an algebraic invariant of the operator $X_{1}$. Then the equation for the first integral is written as

$$
\left.\frac{D_{t}\left(I^{(1)}\right)}{D_{t}(\Phi)}\right|_{20}=\hat{\Theta}\left(I^{(1)}\right)
$$

with some function $\hat{\Theta}$. The integration of this equation provides the first integral of the initial equation $\hat{H}\left(I^{(1)}, \Phi\right)=0$. The obtained equation admits the operator $X_{1}$, therefore, it can be integrated by quadratures.

On the other hand, by the classical method of order reducing, at the first step we choose the operator $X_{1}$ forming an ideal of the admitted algebra $L_{2}$. Then choosing the function $\phi\left(I_{0}\right)$ as the algebraic invariant for $X_{1}$ and the invariant $I^{(1)}$ of the algebra $L_{2}$ as the first order invariant, we obtain a similar reduced first order equation (21). Therefore, we have shown that under an appropriate choice of differential invariants, the classical method of successive order reducing and the method of order reducing by OID lead to the same reduced equation.

The feature of the method of order reducing by OID is that the reduced equation is written in the initial variables and its symmetry is obtained as a linear combination of the initial operators.

Example 2. Consider the equation

$$
\ddot{x}=\frac{\dot{x}}{x^{2}}-\frac{1}{t x}
$$

admitting the operators

$$
X_{1}=t^{2} \frac{\partial}{\partial t}+t x \frac{\partial}{\partial x}, \quad X_{2}=t \frac{\partial}{\partial t}+\frac{x}{2} \frac{\partial}{\partial x}
$$

satisfying the commutation relation $\left[X_{1}, X_{2}\right]=-X_{1}$.

The invariants of the admitted algebra are $I^{(1)}=x \dot{x}-\frac{x^{2}}{t}, I^{(2)}=x^{3} \ddot{x}$. The coefficient OID is found the by the system of equations

$$
X_{1}(\Phi)=0, \quad X_{2}^{(1)}(\Phi)=1
$$


where $\Phi=\Phi(t, x)$. We obtain

$$
\Phi=2 \ln \frac{t}{x}, \quad \lambda D_{t}=\frac{t x}{2(x-t \dot{x})} D_{t}
$$

and applying OID to the invariant $I_{1}$, in view of $(22)$ we get the expression

$$
\frac{\mathrm{d} I^{(1)}}{I^{(1)}+1}=-\frac{1}{2} \mathrm{~d} \Phi .
$$

The integration of this relation leads us to the reduced equation

$$
\frac{x(t \dot{x}-x)}{t}=C_{1} \frac{x}{t}-1
$$

admitting the operator $X_{1}$. It is easy to show that a similar reduced equation is obtained by using the classical method if as the invariants of the operator $X_{1}$ we choose $I_{0}=-2 \ln \frac{x}{t}$ and $I_{1}=x \dot{x}-\frac{x^{2}}{t}$.

\section{System of TWO SECOND ORDER ODES}

We consider the systems

$$
\left\{\begin{array}{l}
\ddot{x}=f(t, x, y, \dot{x}, \dot{y}), \\
\ddot{y}=g(t, x, y, \dot{x}, \dot{y})
\end{array}\right.
$$

admitting four-dimensional Lie algebras of the operators with the basis

$$
X_{i}=\tau_{i}(t, x, y) \frac{\partial}{\partial t}+\xi_{i}(t, x, y) \frac{\partial}{\partial x}+\eta_{i}(t, x, y) \frac{\partial}{\partial y}, \quad i=1, \ldots, 4 .
$$

Suppose that system 24 has the following invariant representation

$$
I_{1}^{(2)}=F(I), \quad I_{2}^{(2)}=G(I)
$$

with some functions $F$ and $G$, where $I$ is a first order differential invariant or an algebraic invariant, and $I_{k}^{(2)}, k=1,2$, are second order differential invariants.

It was shown in work [12 that if system (24) has invariant representation (25), then system (9) always has a non-trivial solution. This seems to be related to the fact that all four-dimensional Lie algebras can be expanded into the direct sum of subalgebras of smaller dimensions, one of which is solvable. This is why for all systems of two second order ODEs with four symmetries admitting invariant representation (25), the reduction to a third order system is possible. If system (9) for the reduced algebra $L_{3}$ has a non-trivial solution, the order of the reduced system can be also reduced. One can show that for all solvable algebras $L_{3}$, system (9) has a non-trivial solution, while for non-solvable algebras it does not. In the latter case the order of the reduced system can not be reduced anymore.

Example 3. Assume that system (24) admits the operators

$$
t \frac{\partial}{\partial t}, \quad x \frac{\partial}{\partial x}, \quad y \frac{\partial}{\partial y}, \quad \frac{\partial}{\partial y} .
$$

Then the invariant representation of the system is of form (25), where

$$
I=\frac{t \dot{x}}{x}, \quad I_{1}^{(2)}=\frac{t^{2} \ddot{x}}{x}, \quad I_{2}^{(2)}=\frac{t \ddot{y}}{\dot{y}} .
$$


The function $\Phi(t, x, y, \dot{x}, \dot{y})$ in OID of form $\left(D_{t} \Phi\right)^{-1} D_{t}$ is determined by the following system of equations

$$
\begin{aligned}
& t \Phi_{t}-\dot{x} \Phi_{\dot{x}}-\dot{y} \Phi_{\dot{y}}=C_{1}, \\
& x \Phi_{x}+\dot{x} \Phi_{\dot{x}}=C_{2}, \\
& y \Phi_{y}+\dot{y} \Phi_{\dot{y}}=C_{3}, \\
& \Phi_{y}=C_{4} .
\end{aligned}
$$

The completeness of this system implies that $C_{4}=0$, while other constants can be arbitrary. Let $C_{1}=-1, C_{2}=1, C_{3}=1$. Then $\Phi=\ln x \dot{y}$, and the reduced system is

$$
\ln x \dot{y}=H\left(I, K_{1}\right), I_{1}^{(2)}=F(I)
$$

with some functions $H$ and $F$, and $K_{1}$ is the integration constant. The reduced system admits the operators

$$
t \frac{\partial}{\partial t}+x \frac{\partial}{\partial x}, \quad x \frac{\partial}{\partial x}-y \frac{\partial}{\partial y}, \quad \frac{\partial}{\partial y} .
$$

For a new OID the function $\Phi$ is determined by the system

$$
\begin{aligned}
& t \Phi_{t}+x \Phi_{x}-\dot{y} \Phi_{\dot{y}}=C_{1}, \\
& x \Phi_{x}-y \Phi_{y}+\dot{x} \Phi_{\dot{x}}-\dot{y} \Phi_{\dot{y}}=C_{2}, \\
& \Phi_{y}=C_{3},
\end{aligned}
$$

and the completeness condition gives $C_{3}=0$, and $C_{1}$ and $C_{2}$ are arbitrary. For instance, $C_{1}=C_{2}=1$. Then $\Phi=\ln x$, the new reduced system is

$$
\frac{t \dot{x}}{x}=H_{1}\left(\ln x, K_{1}, K_{2}\right), \quad x \dot{y}=H_{2}\left(\ln x, K_{1}, K_{2}\right),
$$

where $H_{1}, H_{2}$ are some functions, $K_{i}, i=1,2$, are integration constants and admit the operators

$$
t \frac{\partial}{\partial t}+y \frac{\partial}{\partial y}, \quad \frac{\partial}{\partial y} \text {. }
$$

For these operators the function $\Phi$ in OID is found by the system

$$
t \Phi_{t}+y \Phi_{y}=C_{1}, \quad \Phi_{y}=C_{2},
$$

where the completeness condition implies that $C_{2}=0$. Let $C_{1}=1$, then $\Phi=\ln t$, and system (26) is reduced to

$$
x=Q_{1}\left(\ln t, K_{1}, K_{2}, K_{3}\right), \quad \dot{y}=Q_{2}\left(\ln t, K_{1}, K_{2}, K_{3}\right),
$$

where $Q_{1}, Q_{2}$ are some functions and $K_{i}, i=1,2,3$, are integration constants. This system admits the operator

$$
\frac{\partial}{\partial y}
$$

The function $\Phi$ of a new OID is found by the equation

$$
\Phi_{y}=C_{1} .
$$

Let $C_{1}=1$, then $\Phi=y$ and we obtain the solution to the initial system:

$$
x=Q_{1}\left(\ln t, K_{1}, K_{2}, K_{3}\right), \quad y=\hat{Q}_{2}\left(\ln t, K_{1}, K_{2}, K_{3}, K_{4}\right),
$$

where $Q_{1}, \hat{Q}_{2}$ are some functions and $K_{i}, i=1,2,3,4$, are the integration constants. 


\section{BIBLIOGRAPHY}

1. S. Lie, G. Scheffers. Lectures on differential equations with known infinitesimal transformations. Regular and Chaotic Dynamics, Moscow-Izhevsk (2011). [Vorlesungen über Differentialgleichungen mit bekannten infinitesimalen Transformationen. B. G. Teubner, Leipzig. (1891), (in German).]

2. L.V. Ovsiannikov. Group analysis of differential equations. Nauka, Moscow (1978). [Academic Press, New York (1982).]

3. P.J. Olver. Applications of Lie groups to differential equations. Graduate Texts in Math. 107. Springer, New York (1986).

4. N.H. Ibragimov. Elementary Lie group analysis and ordinary differential equations. Wiley, Chichester (1999).

5. G.W. Bluman, S.C. Anco. Symmetry and integration methods for differential equations. Springer, New-York (2002).

6. Ar. Tresse. Sur les invariants différentiels des groupes continus de transformations // Acta Math. 18:1,1-3 (1894).

7. I.V. Shirokov. Differential invariants of the transformation group of a homogeneous space // Sibir. Matem. Zhurn. 48:6, 1405-1421 (2007). [Siberian Math. J. 48:6, 1127-1140 (2007).]

8. M. M. Goncharovskiy, I. V. Shirokov. Differential invariants and operators of invariant differentiation of the projectable action of Lie groups // Theor. Matem. Fiz. 183:2, 202-221 (2015). [Theor. Math. Phys. 183:2, 619-636 (2015).]

9. R.E. Popovich, V.N. Bojko. Differential invariants of one-parametric group of local transforms and integrable Riccatti equations // Vestnik SamGU. 4(18), 49-56 (2001). (in Russian).

10. O.V. Gaponova, M.O. Nesterenko. Systems of second-order ODEs invariant with respect to lowdimensional Lie algebras // Zb. Pr. Inst. Mat. NAN Ukr. 3:2, 71-91 (2006). (in Ukrainian).

11. M. Ayub, F.M. Mahomed, M. Khan, M.N. Qureshi. Symmetries of second-order systems of ODEs and integrability // Nonlinear Dyn. 74:4, 969-989 (2013).

12. A.A. Gainetdinova, R.K. Gazizov. Integrability of systems of two second-order ordinary differential equations admitting four-dimensional Lie algebras // Proc. Royl. Soc. A. 473:2197, id 20160461 (2017).

13. N.M. Günter. Integration of first order partial differential equations. ONTI, Leningrad-Moscow (1934) (in Russian).

Rafail Kavyevich Gazizov,

Scientific research laboratory

Group analysis of mathematical models

in natural sciences, techniques and technologies

Ufa State Aviation Technical University,

K. Marx str. 12,

450008, Ufa, Russia

E-mail: gazizovrk@gmail.com

Aliya Aidarovna Gainetdinova,

Scientific research laboratory

Group analysis of mathematical models

in natural sciences, techniques and technologies

Ufa State Aviation Technical University,

K. Marx str. 12,

450008, Ufa, Russia

E-mail: gainetdinova.alia@gmail.com 